\title{
PROGRESS IN BRAIN RESEARCH
}

\section{Real-World Applications in Cognitive Neuroscience}

\section{3}

Farahani, J., Soltani, P., Rezlescu, C., \& Walsh, V. (2020). Assessing decision making using 2D animations in elite academy footballers. Progress in Brain Research, 253. doi: 10.1016/bs.pbr.2020.06.016

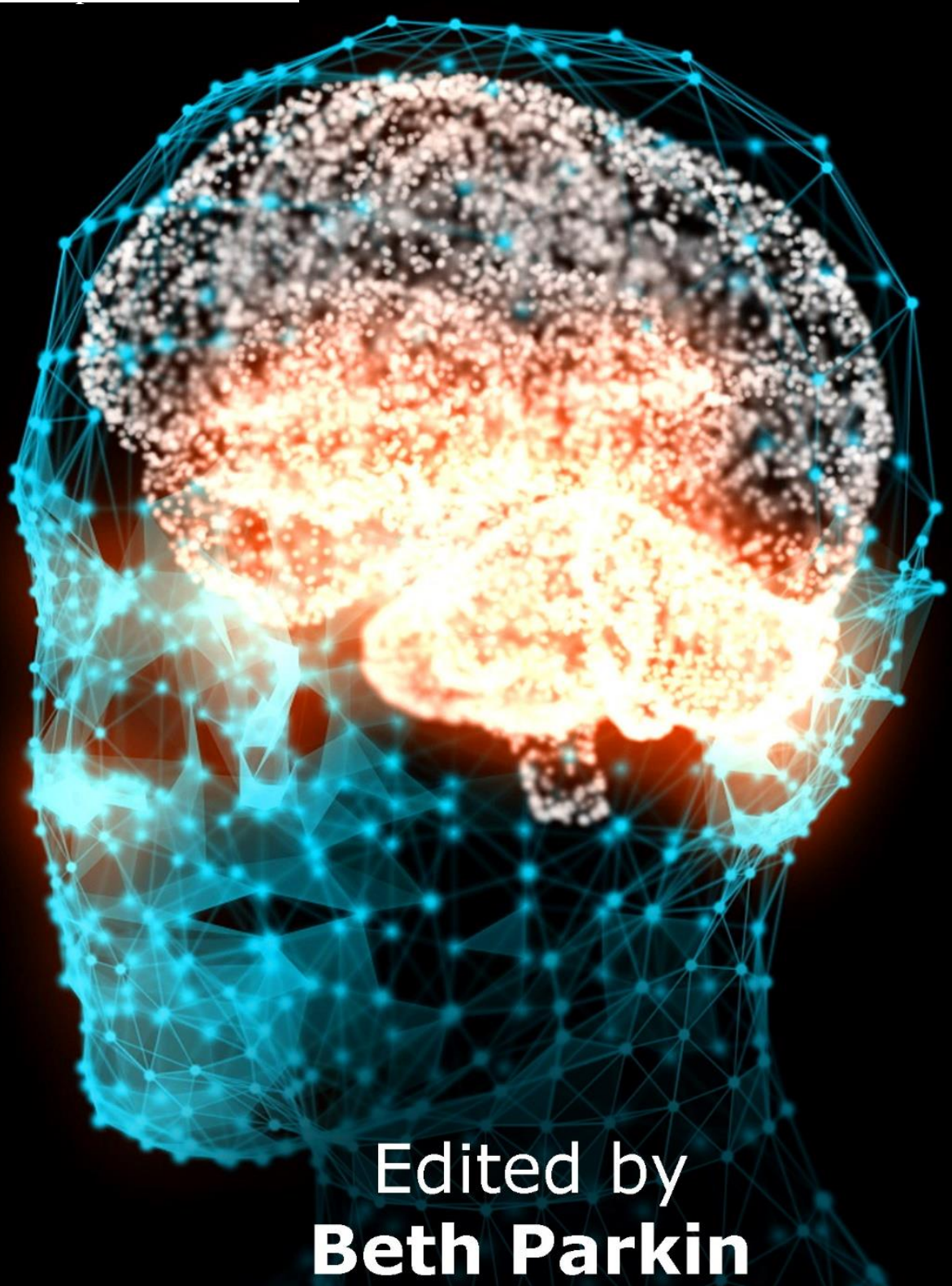




\title{
Assessing decision making using 2D animations in elite academy footballers
}

\author{
Javid Farahani ${ }^{\mathrm{a}}$, Pooya Soltani ${ }^{\mathrm{b}, \mathrm{c}}$, Constantin Rezlescu ${ }^{\mathrm{d}}$, Vincent Walsh ${ }^{\mathrm{a}}$ \\ ${ }^{a}$ Institute of Cognitive Neuroscience, University College London, Alexandra House, \\ Bloomsbury, London, United Kingdom \\ ${ }^{b}$ Centre for the Analysis of Motion, Entertainment Research and Applications (CAMERA), \\ Department of Computer Science, Department of Health, University of Bath, Bath, United \\ Kingdom \\ ${ }^{c}$ Department of Physical Education and Sport Sciences, School of Education and \\ Psychology, Shiraz University, Shiraz, Iran \\ ${ }^{d}$ Department of Experimental Psychology, University College London, London, United \\ Kingdom
}

\begin{abstract}
Having investigated the effects of videos in the preceding chapter, this chapter assesses 2D animation, a form of presentation used in many coaching situations. The aim of this experiment was to investigate decision-making skills in different age groups (Under 16, 18 and 23) of elite academy footballers using a 2D animation simulation task of real game football scenarios. The work also explored the relationship between individual performance on the task and the actual performance on the pitch, as rated by three independent expert football coaches. This allowed us to examine whether this task is useful in predicting real-world decision-making skills. The results suggested that there was a significant difference between age groups on accuracy, by gaining more experience footballers perform better on the task. Also, the results showed a significant difference between all age groups on the response time. The under 23 age group were fastest, then the under 18 age group and finally the under 16 footballers were the slowest on the task. The correlation between performance on the task and the assessments provided by the coaches showed that $2 \mathrm{D}$ animation task is a sensitive measure in assessment of decision-making skills of elite academy players.
\end{abstract}

Keywords: Expertise; Decision-making; Football; Elite athletes; Talent identification

\section{Introduction}

Simulations have been widely used to assess and train perceptual-cognitive skills over the last few decades in highly demanding cognitive domains such as sport (Farahani et al., 2017; Lorains et al., 2013; Soltani, 2017), aviation (Jones et al., 1999; Salas et al., 1998), nursing education (Alinier et al., 2006; Medley and Claydell Horne, 2005; Strayer, 1979), surgical (Hamstra et al., 2006; Satava, 2008; Sutherland et al., 2006) and the military (Aebersold, 2016; Macedonia, 2002; Page and Smith, 1998) due to advantages such as cost-effectiveness, controlling assessment and training environments, and the safety and availability of repeating training.

Scientists have investigated the most important factors contributing to validity and quality of simulation since the very early application of it in both research and real-world environments (e.g., Alessi, 1988; Allen et al., 1986). The most important factor in the effectiveness of this method is related to the degree of fidelity. Allen et al. (1986; p. 497) defined fidelity in simulation as "the degree to which the training devices must duplicate the 
actual environment." Fidelity is not only about the similarity of simulation and physical environment but also, includes equipment fidelity (defined as replication of actual equipment), cognitive fidelity (defined as the degree to which simulation replicates psychological and cognitive factors) and visual-audio fidelity (Kaiser and Schroeder, 2003; Rinalducci, 1996; Zhang, 1993) (defined as replication of visual and auditory stimulus). With current advances in digital technology, there is an increasing trend to using technologies such as animation (e.g., Bai et al., 2016; Hohmann et al., 2016), video (Larkin et al., 2018; Lorains et al., 2013) and virtual reality (Bideau et al., 2009; Tsai et al., 2019; Vignais et al., 2015) to assess and train perceptual-cognitive skills such as anticipation and decision making in both research and elite performance.

To understand the key characteristics of expertise in elite performance, researchers have employed the expert-novice paradigm using the elite performance approach (EPA) proposed by Ericsson and Smith (1991) and have also used 2D animation, video and virtual reality simulation platforms to create tasks which are representative of real-world demands in order to compare elite and novice athletes. The main focus of early research was on the use of video for anticipation and skills in individual and group sports (e.g., Johnson and Raab, 2003; Lorains and MacMahon, 2009; Vaeyens et al., 2007). Most of these studies employed the occlusion paradigm to differentiate the participants. This paradigm alters video footage temporally and spatially and has been used to identify the most important cues that elite participants use based on the "do more with less" concept (e.g., Müller et al., 2006; see Williams and Jackson, 2019 for a more in-depth review of the acquisition of perceptualcognitive skills).

However, the occlusion paradigm has its limitations. In fact, it did not replicate the experience of athletes in a real game scenario (Lorains et al., 2014). Also, the results of more recent studies have suggested that the occlusion paradigm may not be a perfect solution to test perceptual-cognitive skills of expert athletes, as an elite athlete can perform faster when all information is present, in comparison with responding only to the cues (Glöckner and Betsch, 2012). The researchers found that removing information may result in confusion and necessity for a longer response time as participants are trying to "fill in the blanks." Based on these findings, the initial principles of cognitive fidelity proposed by Kaiser and Schroeder (2003) are not met using the occlusion paradigm since in elite performance, both accuracy and response time are equally important to distinguish winners and losers.

Following this research, Lorains et al. (2013) conducted the first research using speed to understand elite versus novices' performance on a video-based task, instead of employing the occlusion paradigm. They compared elite, sub-elite and novice athletes on a video-based task and manipulated the speed of playing a video to enhance the level of fidelity of the assessment. This was similar to previous work in anticipation and decision-making. They found that elite players become more efficient when the speed of a video is played at above real-time speed (faster than normal).

The other limitation of primary research in anticipation using video-based assessments is the focus on one-to-one scenarios in individual actions. For example, there is an extensive number of studies on penalties in soccer (e.g., McMorris and Colenso, 1996; Murgia et al., 2014; Smeeton and Williams, 2012), tie-breaks in tennis (Rowe et al., 2009; Williams et al., 2002), free shots in basketball (Aglioti et al., 2008; de Oliveira et al., 2008), handball (Loffing and Hagemann, 2014; Schorer and Baker, 2009), cricket (Brenton et al., 2016; Müller et al., 2006) and badminton (Jin et al., 2011; Hagemann and Memmert, 2006) but there are fewer studies on tactical decision-making. After reviewing the last 50 years of research on anticipation and decision making, Williams and Jackson (2019) suggested that a distinction be made between reactive short-term decision making and broader tactical decision-making. In a fast-changing environment like soccer, the ability to pick up cues from 
the body language of an athlete is less important in tactical decision-making as they may be far from the position of decision-maker on the pitch, and instead the ability to read the pattern of the team is the key to the game intelligence of players.

Finally, there was a trend toward using big screens instead of computer-based tasks to increase the fidelity of assessment compared to the real game experience, as proposed by Williams and Ericsson (2005). Spittle et al. (2010) investigated the effect of the size of the screen on perceptual-cognitive skills assessment. In their research 72 participants in three groups (elite, sub-elite and novice) took part and were tested on a computer and a video projection screen on basketball tactical decision-making task. Results indicated that screen size has no effect on participants' accuracy on the task, and elite players made better decisions in comparison with a novice on both small and large screens.

The second type of simulation which has received some attention in the last few years is pattern recognition and contextual information when removing visual cues in video presentations. In a study conducted by Murphy et al. (2016) they drew attention to the importance of contextual information instead of postural information from an opponent during an anticipation task. They used players' movement and ball trajectory data to create animation simulation tasks for anticipation of ball bounce location. They compared two groups of participants by video and animation and the results showed skilled players were more accurate than novices in both conditions. They also reported that by using eye-tracking technology, they could show that that elite athletes employed different gaze behaviors, and their verbal reports were indicative of more thorough evaluation of contextual information available. Their findings shed light on the importance of contextual information in anticipation skills. Later on, in the study by Murphy et al. (2019) on the importance of postural and contextual information for expert anticipation in tennis, they reported that few options are generated by participants when postural cues are available. Their findings suggested that anticipation when there is a short distance between the player and opponent, relies on both postural and contextual information.

While some investigations have been conducted to investigate the importance of contextual information in individual sports, few have investigated the specific sources of contextual information employed in team sports such as soccer by using simulation. North et al. (2009) compared elite and novice players on an anticipation and judgment task on both video and point-light simulation and reported that elite participants demonstrated superior anticipation skills than their less-skilled counterparts.

In a follow-up study, North et al. (2016) explored whether anticipation is underpinned by perceiving structured patterns or postural cues using video and point-light displays (converting video of a match to dots on a screen) from a point of view of a camera behind the goal in a soccer match. They compared elite and novice soccer players in video and pointlight display tasks and reported superior performance for elite players in terms of accuracy on both types of information. They also reported better performance on video in comparison with point-light displays suggesting that elite players can use postural cues more efficiently.

There are some important points to consider in this type of research to date. First of all, the focus is on anticipation and not decision-making, which was considered as a primary skill in comparison with pattern recognition and anticipation according to MacMahon and McPherson (2009). Second, when video footage is used to assess anticipation skills, postural cues could affect the decision of the participant. Further when familiar material is used (for instance footage from Premier League games or world cup) knowledge of players' skills and tactical preferences may affect the participant's decision. In this case, participants will be more likely to follow what they expect from the well-known player rather than focusing on the best tactical option. The third point to consider is the angle of the camera and point of view of the participants which is nowhere close to their experience either in the learning 
sessions in the classroom or in game experience. In all studies so far on the use of point-light displays, researchers used the camera angle behind the goal and justified that as the point of view of the central defender but the reality is that it is only possible to capture the point of view of a central defender if a camera installed on his/her head.

To our knowledge, there has been no attempt so far on the use of 2D animation, a form of presentation used in many coaching situations to investigate the decision-making skills of soccer players. With the recent advances in ball and player tracking technology in most European leagues, the raw data of players' movements in a match is available which can now be converted to a 2D animation, very similar to the tactic boards coaches use to explain their ideas to the players before training and matches. Following the previous studies in using video-based assessment for decision making, the primary objective of the current research is to investigate the difference between Under 16, 18 and 23 elite academy soccer players on a 2D animation simulation assessment as measured by decision accuracy in comparison with elite coaches' assessments. The second objective of this study is to compare participants' performance on the task in terms of accuracy and response time with their performance on the pitch as reported by the coaches' opinion. To our knowledge, there is no attempt so far to understand decision-making skills development in elite academy soccer players and there is limited understanding of the correspondence between coaches' opinions on a specific task for a player and their performance on a representative task.

\section{Methods and materials}

\section{Participants}

In total, 118 male participants (range 15-23 years old, mean 18 years old) took part in three groups (U16, U18 and U23). The U23 group comprised 38 footballers with a mean age of 21.2 years $(\mathrm{SD}=1.26)$ who had been training and playing at the Premier League academy level for at least 5 years. The U18 participants were 41 Premier League academy footballers with a mean age of 17.6 years $(\mathrm{SD}=0.57)$ and at least 4 years of experience at the same level. The U16 group were 39 Premier League academy footballers with a mean age of 15.8 years $(\mathrm{SD}=0.48)$ and a minimum of 4 years' experience at Premier League academy level. They were all based at one of two major Premier League football academies. All participants were naive to the purpose of the study. All participants had normal or corrected-to-normal vision. All participants gave their written informed consent in accordance with the Declaration of Helsinki and the guidelines approved by the Ethical Committee of University College London (UCL).

\section{Experimental design}

The study comprised three experimental groups which differed in age and experience. Each participant performed the tasks while seated using an individual tablet in a quiet room. All testing sessions happened in the morning after team breakfast and before the gym and football session on the pitch to prevent the effect of physical fatigue due to exercise. Participants were asked to perform a tablet-based task by watching short video clips of football matches.

The task consisted of two parts. First, they had a warm-up session (comprised of 5 trials) and once they completed the warm-up, actual testing began with 20 clips. The participants were first shown a paused video of a football match with the location of the ball highlighted for $1 \mathrm{~s}$. The 2D animation clip then played for $5 \mathrm{~s}$, after which the screen paused 
again and three locations where the ball could have been passed to were highlighted (see Fig. 1). The participants' task was to indicate the best option in terms of building a good attacking scenario by tapping on their choice of location on the tablet screen. They had $4 \mathrm{~s}$ to make a decision. After $4 \mathrm{~s}$ no response was available and the software moved to the next clips. Options were ordered indicating the best choice (value $=1$ ), an intermediate choice (value $=$ 2 ), and the worst choice (value $=3$ ), failure to respond in $4 \mathrm{~s}$ was categorized as a value 3 decision. To determine the best choices for each scenario, each video clip was watched and independently rated by three UEFA (Union of European Football Associations) A license coaches and only clips on which two or all3 options agreed were used.
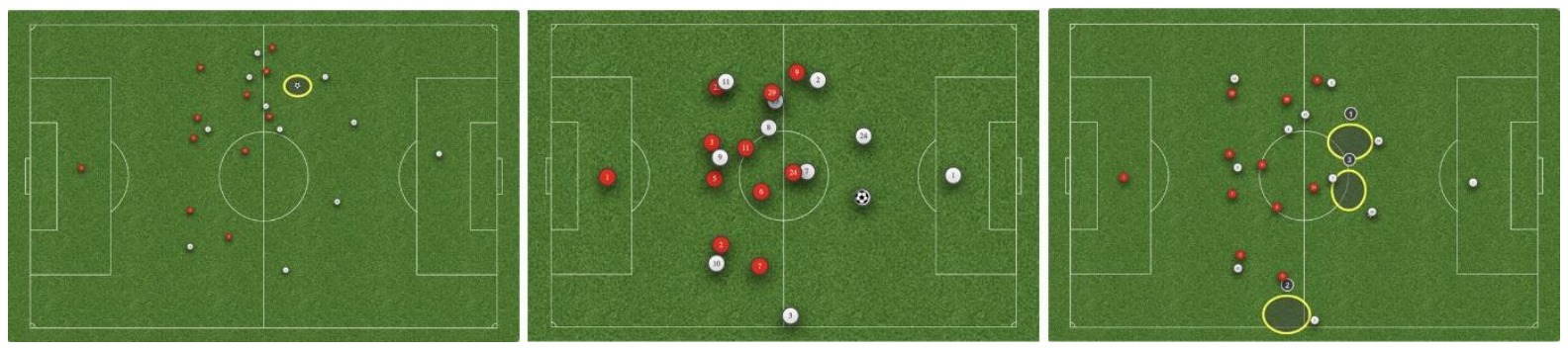

Fig. 1. Diagram showing the order and duration of stimuli for one trial.

The 2D animation clips were extracted from Premier League matches by a camera placed in the middle of the field and converted to birds-eye view. The duration of the 2D animation clips was $5 \mathrm{~s}$ and no feedback was provided during testing sessions (both in the warm-up phase and also in the actual test). Trials were separated by a fixation cross on the screen for $1 \mathrm{~s}$. Participants were asked to respond as quickly and as accurately as possible. Accuracy on the task was measured by converting the value 1-3 to percentage of the maximum possible score, and response time was measured in milliseconds.

The experiment was developed on the Game Intuition ${ }^{\circledR}$ platform (London Cognition limited, UK) using the web portal for editing the 2D animation clips and building the decision-making screen. An iOS tablet pro with 10.5 in. touch (made by Apple Inc., USA) screen was used for the presentation of stimuli and participants were required to tap on the screen of the tablet to indicate their responses.

In order to compare the performance of the participants on the task with how their coaches rated them on the pitch in terms of the judgment of their decision-making skills, a Likert scale of 1-10 was used. Option 1 represented the lowest quality and 10 was the highest. Coaches rated each player on four specific qualities. The first was general decisionmaking (GeneralDM) ability in the game including attacking and defending scenarios. This question covers all the decisions making a player needs to make in a match. The second was on attacking decision-making skills (AttackingDM), which means when a player's team has possession and are going to attack the opponent's goal. This includes the decisions made by players with and without the ball. The third focused on the players' attacking decision-making skills with the ball (DMwBall; excluding attacking moments of the game when the player does not have the ball). The final quality concerned decision-making skills without the ball (DMwoBall; when the player's teammate has the ball and is going to attack the opponent's goal).

\section{Statistical analysis}

One-way analysis of variance (ANOVA) was used to determine if there are any statistically significant differences between the means of the three groups on reaction time and accuracy. To determine which specific groups differed from each other, appropriate post hoc tests are 
carried out. To determine the relationships between accuracy, response time, and coaches assessment of the players' decision making, Spearman's correlation coefficients were used.

\section{Results}

There was a statistically significant difference between groups on accuracy as determined by one-way ANOVA $(\mathrm{F}(2,115)=39.360, \mathrm{P}=0.001)$. A Games-Howell post hoc test revealed that the accuracy was statistically significantly higher in U23 (78.02 \pm 7.34$)$ compared to U18 $(72.34 \pm 4.86, \mathrm{P}=0.001)$ and $\mathrm{U} 16(66.28 \pm 4.94, \mathrm{P}=0.001)$. There was also a statistically significant difference between the U16 and the U18 groups $(\mathrm{P}=0.001)$. There was a statistically significant difference between groups on response time as determined by oneway ANOVA $(\mathrm{F}(2,115)=44.78, \mathrm{P}=0.001)$. A Tukey post hoc test revealed that the response time was statistically significantly lower in U23 $(1.25 \pm 0.58 \mathrm{~ms})$ compared to U18 $(1.91 \pm 0.71 \mathrm{~ms}, \mathrm{P}=0.001)$ and $\mathrm{U} 16(2.78 \pm 0.81 \mathrm{~ms}, \mathrm{P}=0.001)$. There was also a statistically significant difference between the $\mathrm{U} 16$ and the $\mathrm{U} 18$ groups $(\mathrm{P}=0.001)$. 


\section{Correlation between performance on the task and coaches' assessments across all players}

Combining all subjects, accuracy was negatively correlated with RT (rs(118) $=-0.593$, $\mathrm{P}=0.035)$, positively correlated with GeneralDM $(\mathrm{rs}(118)=0.435)$, with AttackingDM $(\operatorname{rs}(118)=0.522)$, with DMwBall $(\operatorname{rs}(118)=0.433)$ and with DMwoBall $(\operatorname{rs}(118)=0.540)$, all for $\mathrm{P}<0.001$. Reaction time was negatively correlated with GeneralDM $(\operatorname{rs}(118)=-0.241)$, with AttackingDM $(\operatorname{rs}(118)=-0.316)$, DMwBall $(\operatorname{rs}(118)=-0.168)$, and with DMwoBall $(\mathrm{rs}(118)=-0.321)$, all for $\mathrm{P}<0.001$. GeneralDM was correlated with AttackingDM $(\operatorname{rs}(118)=0.856)$, with DMwBall $(\operatorname{rs}(118)=0.788)$, and with DMwoBall $(\operatorname{rs}(118)=0.701)$, all for $\mathrm{P}>0.001$. AttackingDM was correlated with DMwBall $(\mathrm{rs}(118)=0.873)$ and DMwoBall $(\mathrm{rs}(118)=0.772)$. DMwBall was also correlated with DMwoBall $(\operatorname{rs}(118)=0.727, \mathrm{P}<0.001)($ Fig. 2$)$.
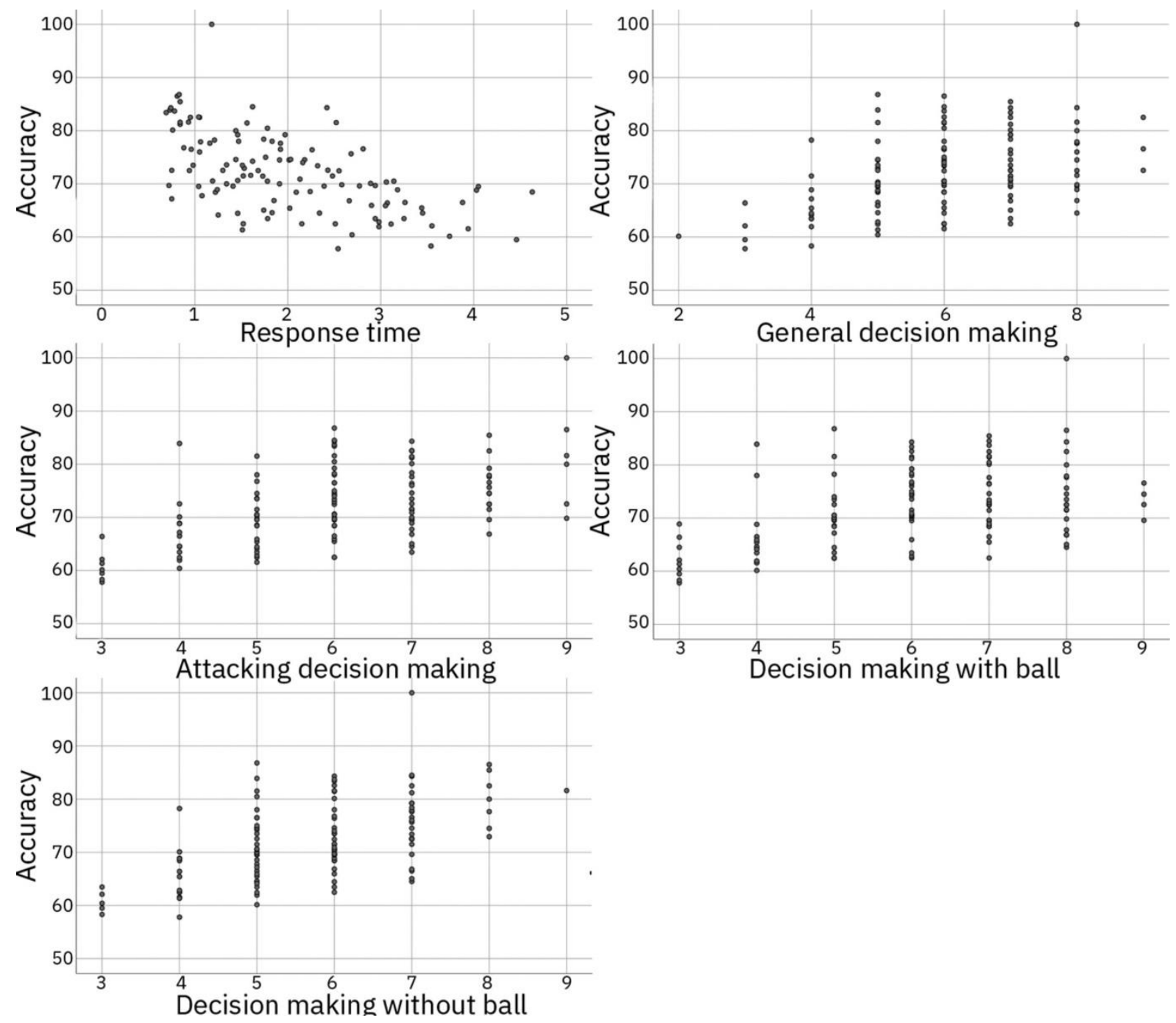

Fig. 2. Scatterplot of correlation of accuracy with RT, GeneralDM, AttackingDM, DMwBall, and DMwoBall for all subjects. 


\section{Correlation between performance on the task and coaches assessment in the U16 group}

For U16 group, accuracy was negatively correlated with RT $\left(\mathrm{r}_{\mathrm{s}}(39)=-0.363, \mathrm{P}=0.23\right)$, positively correlated with GeneralDM $\left(\mathrm{r}_{\mathrm{s}}(39)=0.535\right)$, with AttackingDM $\left(\mathrm{r}_{\mathrm{s}}(39)=0.660\right)$, DMwBall $\left(r_{s}(39)=0.675\right)$, and with DMwoBall $\left(r_{s}(39)=0.585\right)$, all for $\mathrm{P}<0.001$. RT was negatively correlated with AttackingDM $\left(\mathrm{r}_{\mathrm{s}}(39)=-0.379, \mathrm{P}=0.001\right)$ and with DMwBall $\left(r_{s}(39)=-0.390, P=0.014\right)$. GeneralDM was correlated with AttackingDM $\left(r_{s}(39)=0.936\right)$, with DMwBall $\left(r_{s}(39)=0.865\right)$, and with DMwoBall $\left(r_{s}(39)=0.704\right)$, all for $\mathrm{P}<0.001$. AttackingDM was correlated with DMwBall $\left(\mathrm{r}_{\mathrm{s}}(39)=0.935\right)$ and with DMwoBall $\left(\mathrm{r}_{\mathrm{s}}(39)=0.769\right)$, both for $\mathrm{P}<0.001$. DMwBall was also correlated with DMwoBall $\left(\mathrm{r}_{\mathrm{s}}(39)=0.777, \mathrm{P}<0.001\right)($ Fig. 3).
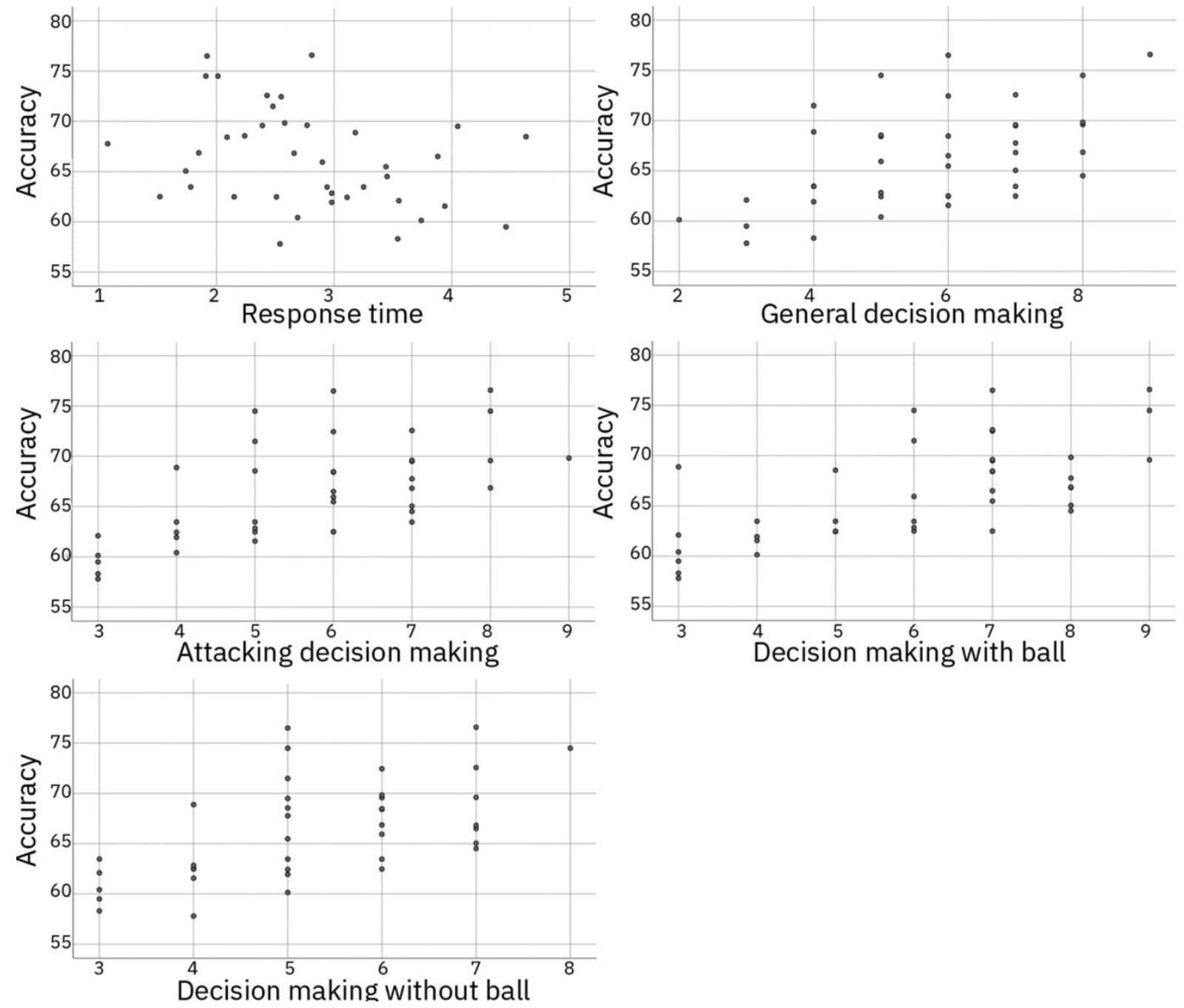

Fig. 3. Scatterplot of correlation of accuracy with RT, GeneralDm, AttackingDM, DMwBall, and DMwoBall in U16. 
For U18 group, accuracy correlated with GeneralDM $\left(\mathrm{r}_{\mathrm{s}}(41)=0.467, \mathrm{P}=0.002\right)$,

AttackingDM $\left(\mathrm{r}_{\mathrm{s}}(41)=0.595\right)$, with DMwBall $\left(\mathrm{r}_{\mathrm{s}}(41)=0.660\right)$, and with DMwoBall $\left(\mathrm{r}_{\mathrm{s}}(41)=0.592\right)$, all for $\mathrm{P}<0.001$. Reaction time was negatively correlated with GeneralDM $\left(\mathrm{r}_{\mathrm{s}}(41)=-0.412, \mathrm{P}=0.007\right)$, with AttackingDM $\left(\mathrm{r}_{\mathrm{s}}(41)=-0.417, \mathrm{P}=0.007\right)$, and with DMwBall $\left(\mathrm{r}_{\mathrm{s}}(41)=-0.332, \mathrm{P}=0.034\right)$. GeneralDM was correlated with AttackingDM $\left(r_{s}(41)=0.817\right)$, with DMwBall $\left(r_{s}(41)=0.780\right)$, and with DMwoBall $\left(r_{s}(41)=0.620\right)$, all for $\mathrm{P}<0.001$. AttackingDM was also correlated with DMwBall $\left(\mathrm{r}_{\mathrm{s}}(41)=0.932\right)$ and with DMwoBall $\left(r_{s}(41)=0.777\right)$, both for $P=0.001$. DMwBall was also correlated with DMwoBall $\left(\mathrm{r}_{\mathrm{s}}(41)=0.857, \mathrm{P}<0.001\right)($ Fig. 4$)$.
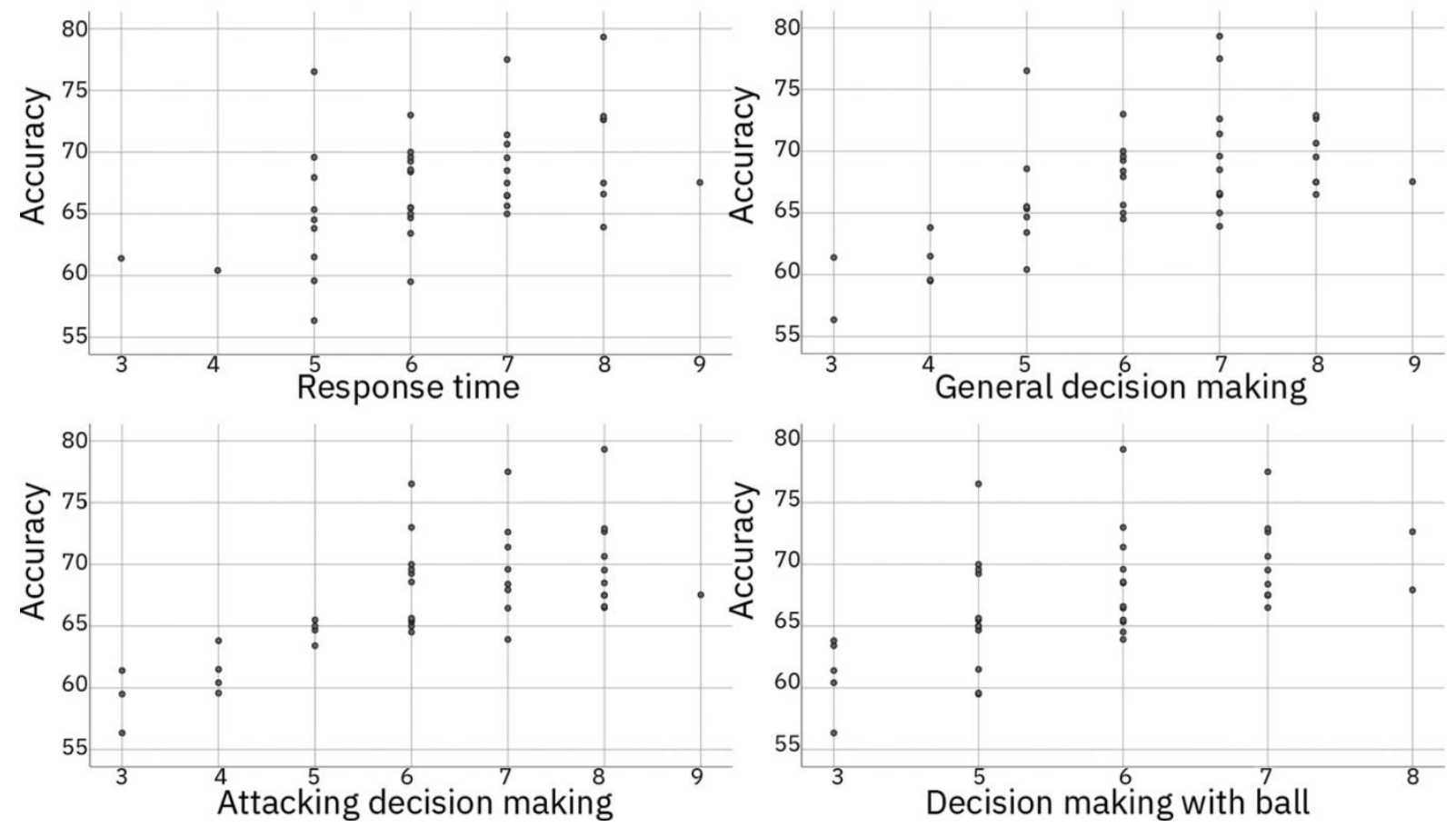

Fig. 4. Scatterplot of correlation between accuracy and GeneralDM, AttackingDM, DMwBall, and DMwoBall for U18. 


\section{Correlation between performance on the task and coaches assessment in the U23 group}

For U23 group, accuracy was negatively correlated with $\mathrm{RT}\left(\mathrm{r}_{\mathrm{s}}(38)=-0.359, \mathrm{P}=0.027\right)$, and positively correlated GeneralDM $\left(\mathrm{r}_{\mathrm{s}}(38)=0.501\right)$, with Attacking DM $\left(\mathrm{r}_{\mathrm{s}}(38)=0.547\right)$, with DMwBall $\left(r_{\mathrm{s}}(38)=0.617\right)$ and with DMwoBall $\left(\mathrm{r}_{\mathrm{s}}(38)=0.466\right)$, all for $\mathrm{P}=0.001$.

GeneralDM was correlated with AttackingDM $\left(\mathrm{r}_{\mathrm{s}}(38)=0.807\right)$, with DMwBall $\left(\mathrm{r}_{\mathrm{s}}(38)=0.766\right)$, and with DMwoBall $\left(\mathrm{r}_{\mathrm{s}}(38)=0.806\right)$, all for $\mathrm{P}<0.001$. AttackingDM was also correlated with DMwBall $\left(\mathrm{r}_{\mathrm{s}}(38)=0.794\right)$ and with DMwoBall $\left(\mathrm{r}_{\mathrm{s}}(38)=0.784\right)$, both for $\mathrm{P}<0.001$. Finally, DMwBall was correlated with DMwoBall $\left(\mathrm{r}_{\mathrm{s}}(38)=0.716, \mathrm{P}<0.001\right)$ (Fig. $5)$.
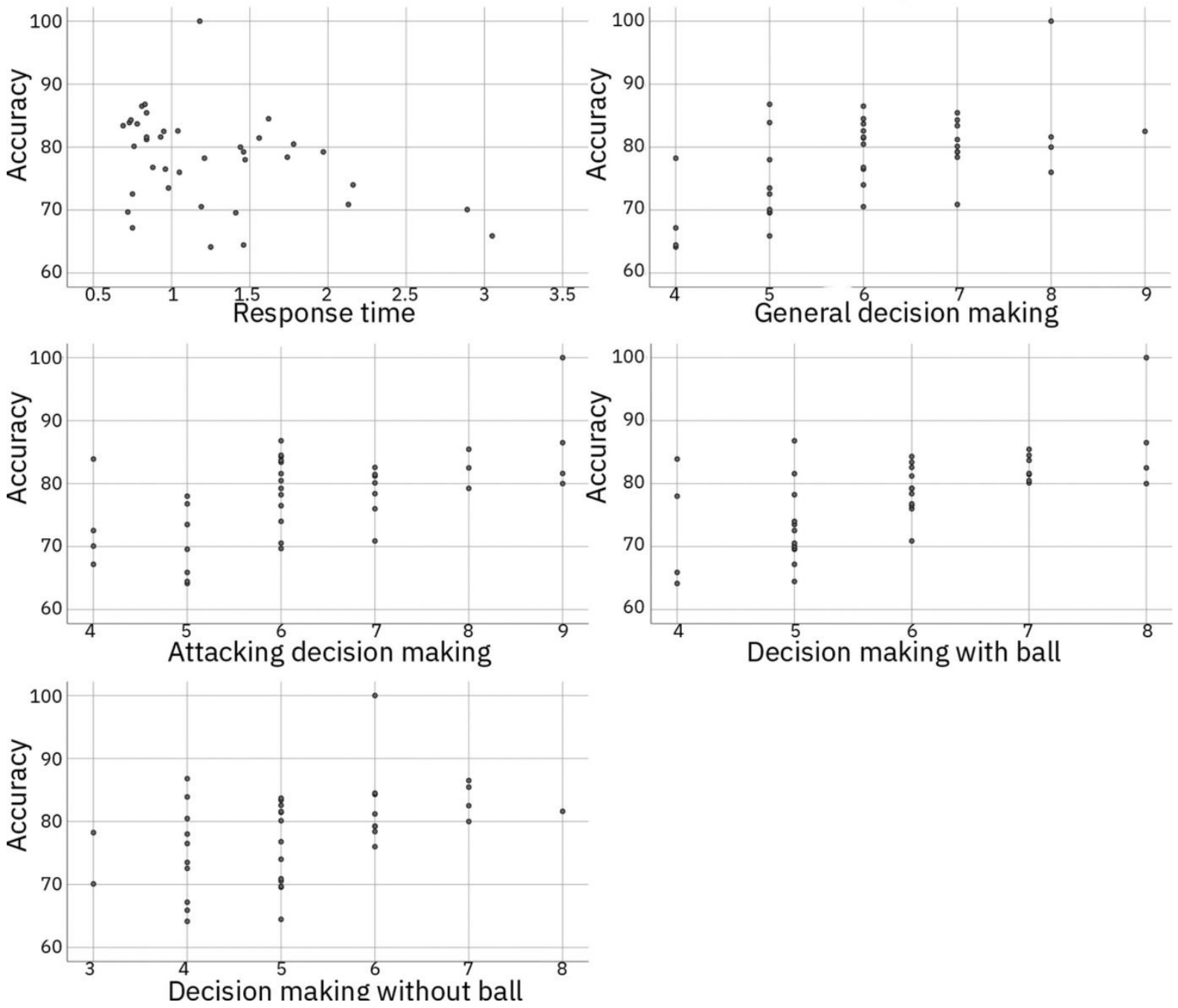

Fig. 5. Scatterplot of correlations of accuracy with RT, GeneralDM, AttackingDM, DMwBall, and DMwoBall for U23.

To summarize the results decision making of all scenarios was positively correlate showed improvements over time. Encouragely the objective data was correlated with the coaches assessments. 


\section{Discussion}

To our knowledge, this is the first experiment to examine expertise development in elite footballers specifically employing 2D animation decision-making. The aim of this experiment was to investigate if there were differences in decision-making skills between different age groups (Under 16, 18 and 23) of elite academy footballers. It also explored the relationship between individual performance on the task and the performance of the footballers on the pitch, as rated by three independent expert football coaches. This allowed us to examine whether this task is useful in predicting real-world decision-making skills. The results suggested that there was a significant difference between age groups on accuracy. By gaining more experience, footballers perform better on the task. Also, the results showed a significant difference between all age groups on the response time. The U23 group were the fastest group, then the U18 and finally the U16 footballers were the slowest on the task. The correlation between performance on the task and assessment of the coaches showed that 2D animation is a sensitive representative task for the assessment of decision-making skills of elite academy players.

In the previous chapter which used video-based assessment, we found no differences between age groups on accuracy of the task. One might think that removing information is going to impair decision-making and be less sensitive. However, part of the thinking here was inspired by video-game history in which realistic simulations are very useful. Also, completely unrealistic simulations are very useful too, but in between, there is an uncanny valley where information that is part real and part not real can actually be distracting. In this experiment, what we discovered is that by simplifying the data we can actually see things in our experiment that we did not with the video-based assessment. For example, in accuracy there were differences between all groups.

To use a musical comparison analogy this maybe similar to considering the difference between a student who can perhaps improvise music but does not know the theory and scales behind the music. By removing the video information, what we are really testing here is the conceptual understanding of the game of the players. Of course, whether that conceptual understanding transfers to the pitch is another question but still remains interesting that this is a way to analyzing their understanding. From a conceptual point of view, it is quite clear that 2D reveals more about the players understanding than video. In regards to players' performance on the tasks in terms of response time, it seems that with more experience, players are also faster at reading the scenario on the task. This allows us to separate the three groups in a more sensitive way than with the video.

In terms of the relationship between players' performance on the task and coaches' assessments of players, similar findings to the previous chapter were observed. Having a statistically positive correlation, general decision-making assessments of coaches with accuracy on the task is an encouraging result as this suggests that the coaches have good insight into what the players can actually do.

It is quite clear that because of the organization of the data (reaction time decrease with age) and because of improved correlation between coaches assessment of the players decision-making and U23 versus U18 performance on the task, that there is clearly something serious and real being achieved in academy football. The challenge now is how we can improve on that in future assessment and training. 


\section{References}

Aebersold, M., 2016. The history of simulation and its impact on the future. AACN Adv. Crit. Care 27 (1), 56-61.

Aglioti, S.M., Cesari, P., Romani, M., Urgesi, C., 2008. Action anticipation and motor resonance in elite basketball players. Nat. Neurosci 11 (9), 1109.

Alessi, S.M., 1988. Fidelity in the design of instructional simulations. J. Comput. Based Instr. 15 (2), 40-47.

Alinier, G., Hunt, B., Gordon, R., Harwood, C., 2006. Effectiveness of intermediate-fidelity simulation training technology in undergraduate nursing education. J. Adv. Nurs. 54 (3), 359-369.

Allen, J.A., Hays, R.T., Buffardi, L.C., 1986. Maintenance training simulator fidelity and individual differences in transfer of training. Hum. Factors 28 (5), 497-509.

Bai, Y., Kaufman, D.M., Liu, C.K., Popovi_c, J., 2016. Artist-directed dynamics for 2D animation. ACM Trans. Graph. (TOG) 35 (4), 145.

Bideau, B., Kulpa, R., Vignais, N., Brault, S., Multon, F., Craig, C., 2009. Using virtual reality to analyze sports performance. IEEE Comput. Graph. Appl. 30 (2), 14-21.

Brenton, J., Muller, S., Mansingh, A., 2016. Discrimination of visual anticipation in skilled cricket batsmen. J. Appl. Sport Psychol. 28 (4), 483-488.

de Oliveira, R.F., Oudejans, R.R., Beek, P.J., 2008. Gaze behavior in basketball shooting: further evidence for online visual control. Res. Q. Exerc. Sport 79 (3), 399-404.

Ericsson, K.A., Smith, J. (Eds.), 1991. Toward a General Theory of Expertise: Prospects and Limits. Cambridge University Press.

Farahani, J.J., Javadi, A.H., O’Neill, B.V., Walsh, V., 2017. Effectiveness of above real-time training on decision-making in elite football: a dose-response investigation. In: Progress in Brain Research. Vol. 234. Elsevier, pp. 101-116.

Glockner, A., Betsch, T., 2012. Decisions beyond boundaries: when more information is processed faster than less. Acta Psychol. 139 (3), 532-542.

Hagemann, N., Memmert, D., 2006. Coaching anticipatory skill in badminton: laboratory versus field-based perceptual training. J. Human Movement Stud. 50 (6), 381-398.

Hamstra, S.J., Dubrowski, A., Backstein, D., 2006. Teaching technical skills to surgical residents: a survey of empirical research. Clin. Orthop. Relat. Res. 449, 108-115.

Hohmann, T., Obeloer, H., Schlapkohl, N., Raab, M., 2016. Does training with 3D videos improve decision-making in team invasion sports? J. Sports Sci. 34 (8), 746-755.

Jin, H., Xu, G., Zhang, J.X., Gao, H., Ye, Z., Wang, P., Lin, H., Mo, L., Lin, C.D., 2011. Eventrelated potential effects of superior action anticipation in professional badminton players. Neurosci. Lett. 492 (3), 139-144.

Johnson, J.G., Raab, M., 2003. Take the first: option-generation and resulting choices. Organ. Behav. Hum. Decis. Process. 91 (2), 215-229.

Jones, R.M., Laird, J.E., Nielsen, P.E., Coulter, K.J., Kenny, P., Koss, F.V., 1999. Automated intelligent pilots for combat flight simulation. AI Magazine 20 (1), 27.

Kaiser, M.K., Schroeder, J.A., 2003. Flights of fancy: the art and science of flight simulation. In: Tsang, P.S., Vidulich, M.A. (Eds.), Principles and Practice of Aviation Psychology. Publication of Lawrence Erlbaum Associates, Incorporated, pp. 435-467.

Larkin, P., Mesagno, C., Berry, J., Spittle, M., Harvey, J., 2018. Video-based training to improve perceptual-cognitive decision-making performance of Australian football umpires. J. Sports Sci. 36 (3), 239-246.

Loffing, F., Hagemann, N., 2014. Skill differences in visual anticipation of type of throw in team-handball penalties. Psychol. Sport Exerc. 15 (3), 260-267.

Lorains, M., MacMahon, C., 2009. Adapting the functional overreaching principle to cognitive skills: expertise differences using speed manipulations in a video-based 
decision-making task. In: Proceedings of the 12th World Congress of Sport Psychology (ISSP). Morocco: Marrakesh.

Lorains, M., Ball, K., MacMahon, C., 2013. Expertise differences in a video decision-making task: speed influences on performance. Psychol. Sport Exerc. 14 (2), 293-297.

Lorains, M., Panchuk, D., Ball, K., MacMahon, C., 2014. The effect of an above real time decision-making intervention on visual search behaviour. Int. J. Sports Sci. Coach. 9 (6), 1383-1392.

Macedonia, M., 2002. Games, simulation, and the military education dilemma. In: Internet and the University: 2001 Forum. Educause, Louisville, CO, pp. 157-167.

MacMahon, C., McPherson, S.L., 2009. Knowledge base as a mechanism for perceptual cognitive tasks: skill is in the details!. Int. J. Sport Psychol. 40 (4), 565-579.

McMorris, T., Colenso, S., 1996. Anticipation of professional soccer goalkeepers when facing right-and left-footed penalty kicks. Percept. Mot. Skills 82 (3), 931-934.

Medley, C.F., Claydell Horne, R.N., 2005. Using simulation technology for undergraduate nursing education. J. Nurs. Educ. 44 (1), 31.

Muller, S., Abernethy, B., Farrow, D., 2006. How do world-class cricket batsmen anticipate a bowler's intention? Q. J. Exp. Psychol. 59 (12), 2162-2186.

Murgia, M., Sors, F., Muroni, A.F., Santoro, I., Prpic, V., Galmonte, A., Agostini, T., 2014. Using perceptual home-training to improve anticipation skills of soccer goalkeepers. Psychol. Sport Exerc. 15 (6), 642-648.

Murphy, C.P., Jackson, R.C., Cooke, K., Roca, A., Benguigui, N., Williams, A.M., 2016. Contextual information and perceptual-cognitive expertise in a dynamic, temporally constrained task. J. Exp. Psychol. Appl. 22 (4), 455.

Murphy, C.P., Jackson, R.C., Williams, A.M., 2019. Informational constraints, option generation, and anticipation. Psychol. Sport Exerc. 41, 54-62.

North, J.S., Williams, A.M., Hodges, N., Ward, P., Ericsson, K.A., 2009. Perceiving patterns in dynamic action sequences: investigating the processes underpinning stimulus recognition and anticipation skill. Appl. Cogn. Psychol. 23 (6), 878-894.

North, J.S., Hope, E., Williams, A.M., 2016. The relative importance of different perceptual cognitive skills during anticipation. Hum. Mov. Sci. 49, 170-177.

Page, E.H., Smith, R., 1998. Introduction to military training simulation: a guide for discrete event simulationists. In: 1998 Winter Simulation Conference. Proceedings (Cat. No. 98CH36274). vol. 1. IEEE, pp. 53-60.

Rinalducci, E.J., 1996. Characteristics of visual fidelity in the virtual environment. Presence Teleop. Virt. Environ. 5 (3), 330-345.

Rowe, R., Horswill, M.S., Kronvall-Parkinson, M., Poulter, D.R., McKenna, F.P., 2009. The effect of disguise on novice and expert tennis players' anticipation ability. J. Appl. Sport Psychol. 21 (2), 178-185.

Salas, E., Bowers, C.A., Rhodenizer, L., 1998. It is not how much you have but how you use it: toward a rational use of simulation to support aviation training. Int. J. Aviation Psychol. 8 (3), 197-208.

Satava, R.M., 2008. Historical review of surgical simulation-a personal perspective. World J. Surg. 32 (2), 141-148.

Schorer, J., Baker, J., 2009. An exploratory study of aging and perceptual-motor expertise in handball goalkeepers. Exp. Aging Res. 35 (1), 1-19.

Smeeton, N.J., Williams, A.M., 2012. The role of movement exaggeration in the anticipation of deceptive soccer penalty kicks. Br. J. Psychol. 103 (4), 539-555.

Soltani P., Virtual Swimming: A Psycho-Biophysical Evaluation of an Active Video Game, 2017, Ph.D. Thesis, University of Porto. Available at: https://hdl.handle.net/10216/102743 (Accessed: 12 July 2020). 
Spittle, M., Kremer, P., Hamilton, J., 2010. The effect of screen size on video-based perceptual decision making tasks in sport. Int. J. Sport Exerc. Psychol. 8 (4), 360-372.

Strayer, P.R., 1979. The Effect of Simulation Versus Traditional Instructional Strategies on the Achievement and Attitude of Baccalaureate Nursing Students. The Ohio State University. Doctoral dissertation.

Sutherland, L.M., Middleton, P.F., Anthony, A., Hamdorf, J., Cregan, P., Scott, D., Maddern, G.J., 2006. Surgical simulation: a systematic review. Ann. Surg. 243 (3), 291.

Tsai, W.L., Su, L.W., Ko, T.Y., Yang, C.T., Hu, M.C., 2019. Improve the decision-making skill of basketball players by an action-aware VR training system. In: 2019 IEEE Conference on Virtual Reality and 3D User Interfaces (VR), March. IEEE, pp. 11931194.

Vaeyens, R., Lenoir, M., Williams, A.M., Mazyn, L., Philippaerts, R.M., 2007. The effects of task constraints on visual search behavior and decision-making skill in youth soccer players. J. Sport Exerc. Psychol. 29 (2), 147-169.

Vignais, N., Kulpa, R., Brault, S., Presse, D., Bideau, B., 2015. Which technology to investigate visual perception in sport: video vs. virtual reality. Hum. Mov. Sci. 39, $12-26$.

Williams, A.M., Ericsson, K.A., 2005. Perceptual-cognitive expertise in sport: some considerations when applying the expert performance approach. Hum. Mov. Sci. 24 (3), 283-307.

Williams, A.M., Jackson, R.C., 2019. Anticipation in sport: fifty years on, what have we learned and what research still needs to be undertaken? Psychol. Sport Exerc. 42, 1624.

Williams, A.M., Ward, P., Knowles, J.M., Smeeton, N.J., 2002. Anticipation skill in a real world task: measurement, training, and transfer in tenni. J. Exp. Psychol. Appl. 8 (4), 259.

Zhang, B., 1993. How to consider simulation fidelity and validity for an engineering simulator. In Flight Simulation and Technologies (p. 3598), American institute of aeronautics and astronautics, Inc. 